Hydrol. Earth Syst. Sci., 10, 485-494, 2006

www.hydrol-earth-syst-sci.net/10/485/2006/

(C) Author(s) 2006. This work is licensed

under a Creative Commons License.

\title{
Clustering of heterogeneous precipitation fields for the assessment and possible improvement of lumped neural network models for streamflow forecasts
}

\author{
N. Lauzon ${ }^{1}$, F. Anctil ${ }^{2}$, and C. W. Baxter ${ }^{3}$ \\ ${ }^{1}$ Golder Associates, Burnaby, BC, Canada \\ ${ }^{2}$ Département de génie civil, Pavillon Pouliot, Université Laval, Québec, G1K 7P4, Canada \\ ${ }^{3}$ HYDRANNT Consulting Inc., Port Coquitlam, Canada
}

Received: 20 December 2005 - Published in Hydrol. Earth Syst. Sci. Discuss.: 23 January 2006

Revised: 30 May 2006 - Accepted: 9 June 2006 - Published: 7 July 2006

\begin{abstract}
This work addresses the issue of better considering the heterogeneity of precipitation fields within lumped rainfall-runoff models where only areal mean precipitation is usually used as an input. A method using a Kohonen neural network is proposed for the clustering of precipitation fields. The evaluation and improvement of the performance of a lumped rainfall-runoff model for one-day ahead predictions is then established based on this clustering. Multilayer perceptron neural networks are employed as lumped rainfallrunoff models. The Bas-en-Basset watershed in France, which is equipped with 23 rain gauges with data for a 21 year period, is employed as the application case. The results demonstrate the relevance of the proposed clustering method, which produces groups of precipitation fields that are in agreement with the global climatological features affecting the region, as well as with the topographic constraints of the watershed (i.e., orography). The strengths and weaknesses of the rainfall-runoff models are highlighted by the analysis of their performance vis-à-vis the clustering of precipitation fields. The results also show the capability of multilayer perceptron neural networks to account for the heterogeneity of precipitation, even when built as lumped rainfallrunoff models.
\end{abstract}

\section{Introduction}

Lumped rainfall-runoff models, as opposed to distributed ones, continue to constitute a viable solution for the operational needs of estimating flows in watersheds. They are relatively easy to operate, have low computing requirements, and can provide quick and reasonably accurate estimations at the watershed outlet. Such models are expected to be widely used well into the future. This paper proposes a

Correspondence to: F. Anctil

(francois.anctil@gci.ulaval.ca) method to better analyze one of the shortfalls of lumped hydrological models, which is that heterogeneous precipitation over a watershed cannot not be considered. Indeed, only the mean areal precipitation is usually considered as an input to lumped models, unless a specific subdivision of the watershed can be made and accommodated by the model. In their review of rainfall-runoff models, Singh and Woolhiser (2002) stress the effect of the spatial variability of precipitation on the production of streamflow in a watershed, and this effect has been a long standing issue in hydrology as demonstrated in the work of Naden (1992), and Faures et al. (1995). Dawdy and Bergman (1969), and Wilson et al. (1979) indicate that errors in the estimation of rainfall intensity are very likely to limit the accuracy of rainfall-runoff models, and this would be particularly prevalent for lumped models.

This study involves the use of a clustering algorithm based on the Kohonen neural network for discriminating daily precipitation fields in a watershed into coherent groups. The performance of lumped multilayer perceptron neural network models for the estimation of streamflow on this watershed is afterward assessed with respect to each of the identified groups of precipitation fields. Through this work, three issues are addressed: 1) the relevance of the clustering algorithm for the discrimination of precipitation fields from day to day, 2) the value of evaluating the performance of rainfallrunoff models with respect to precipitation field groups, and 3 ) the possibility of improving rainfall-runoff modelling performance through more specific identification of inputs as highlighted by the clustering.

In terms of precipitation field clustering, any technique may be appropriate. For meteorological data in general, and for precipitation in particular, there is a large range of clustering algorithms that have been employed. The simplest cases involve subjective inferences based on observations on synoptic maps (Bardossy and Plate, 1992; Siew-Yan-Yu et al., 1998). More objective methods normally include one simple discrimination rule such as the Euclidian distance between

Published by Copernicus GmbH on behalf of the European Geosciences Union. 
the features of two events (Shoof and Pryor, 2001) or a probabilistic criterion (Benzie et al., 1997). The level of objectivity can then be increased by including several discrimination rules, as is the case with the Classification and Regression Trees (CART) employed by Hughes et al. (1993), Zorita et al. (1995), and Shnur and Lettenmaier (1998). For the assignation of rules, the work of Bardossy et al. (1994) and Ozelkan et al. (1996) make use of fuzzy logic, which is an alternative to clustering methods with fixed rules. Even the most objective methods contain some level of subjectivity that may induce some uncertainty on the validity of the generated clustering. It must be noted as well that computing requirements increase as the number of rules increases, particularly with large databases (Zorita and Storch, 1999). The Kohonen neural network employed as the clustering algorithm in this study possesses some amount of subjectivity. However, its process for the determination of clusters (i.e., calibration of the Kohonen network) may be less demanding in terms of computing requirements than more traditional and common clustering techniques, such as those presented in Dillon and Goldstein (1984), as shown in a large clustering study by Lauzon (2003).

Multilayer perceptron neural networks are employed here as rainfall-runoff models. They have been widely acknowledged as being appropriate for rainfall-runoff modelling (ASCE, 2000a and b; Singh and Woolhiser, 2002), and such lumped models are easy to build and implement on an operational basis. They are employed here for one-day ahead streamflow forecasts. Other noteworthy applications of neural networks include short-term rainfall prediction for realtime flood forecasting (Toth et al., 2000; Brath et al., 2002) and output updating of deterministic lumped rainfall-runoff forecasting models (Brath et al., 2002; Anctil et al., 2003).

The selection of an appropriate study watershed is essential to achieve the objectives of this work. In order to produce variability in the areal precipitation estimates, the watershed must have heterogeneous precipitation fields. For the same reason, the rain gauge network must include a large number of stations.

In the following section of this paper, a brief description of the Kohonen and multilayer perceptron neural networks, used for the clustering of precipitation fields and rainfallrunoff modelling, is given. In subsequent sections, the context of application, including the description of the Bas-enBasset (France) watershed and its database, as well as the details on the experimental protocol, is presented. This is followed by the analysis of the results, with an emphasis on the issues of interest: 1) the relevance of the clustering algortithm, 2) the analysis in rainfall-runoff modelling performance based on the clustering, and 3) the improvement of modelling performance. Finally, a conclusion highlighting the relevant findings is presented.

\section{Description of the neural networks}

\subsection{Descriptive neural networks}

The structure of a Kohonen neural network is designed so as to identify patterns in data and as such can be used as a clustering technique. This network is a descriptive tool that is used increasingly in hydrology and water resources, in applications such as the clustering of watershed conditions ( $\mathrm{Li}$ ong et al., 2000); the determination of hydrological homogeneous regions (Hall and Minns, 1999); the identification of river pollutant sources (Gotz et al., 1998); and the study of algae bloom (Bowden et al., 2002). Like any clustering technique, the Kohonen network requires that the data domain under study be divided in terms of input vectors (e.g., the historical record of precipitation from several stations at a given day, for each day). The network is made of an input layer of neurons that receives the data and an output layer often structured in a planar surface. Each input neuron in the output layer is connected to each output neuron in the output layer, and a weight is assigned to each connection. An input vector is described as $I=I_{1}, I_{2}, \ldots, I_{n}$, where $n$ is the number of neurons in the input layer. The weight vector attached to output neuron $j$ through the connections with the input layer is defined as $W_{j}=W_{1, j}, W_{2, j}, \ldots, W_{n, j}$, where $n$ is again the number of neurons in the input layer. The weight vector of each output neuron is of the same dimension as the input vector, and consequently can be considered as a mass center of a cluster. Any input vector given to the network is compared with the weight vector of each output neuron. A neuron is said to be activated (i.e. chosen) when its weight vector is the closest in distance to the input vector given to the network.

The elements of all the weight vectors must be calibrated so as to cover the whole data domain. The calibration is an iterative process, where one input vector is fed to the network at a time (i.e. iteration). Following the feeding of an input vector $I$ at a given iteration, the weight vector $W_{j}$ of each of the output neurons is updated as follows (Kohonen, 1990):

$$
W_{j}^{(t)}=W_{j}^{(t-1)}+h_{j}\left(I-W_{j}^{(t-1)}\right)
$$

This formulation simply drives the weight vector to be closer to the input vector. For the description of precipitation fields performed in this work, the term $h_{j}$ is expressed as:

$h_{j}=h_{0} \exp \left(-\left(d_{j, a} / \sigma\right)^{2}\right)$

Equation (2) is a standard form that is regularly used with the Kohonen network for the study of natural phenomena (Kohonen, 1990). In this equation, $d_{j, a}$ is the Euclidian distance between the most suitable output neuron $a$ and another output neuron $j$, as determined by the position of the output neurons on the output map (layer). When $j=a$, the exponential equals 1 (i.e. distance equals 0 ) and the value of $h_{j}$ is at 
its maximum value (i.e. $h_{0}$ ). The value of $h_{j}$ decreases as the distance between activated neuron $a$ and neuron $j$ increases. Parameter $h_{0}$ gives the magnitude of the updating applied to the weights, while parameter $\sigma$ is a scaling factor that indicates the extent of the output map affected by the updating. Both parameters are set at a high value at the start of the calibration process to ensure a rapid spreading of the output neurons over the data domain, and are reduced gradually so that only small adjustments are performed at the end of the calibration process. A large number of iterations ensures that all available input vectors are employed a significant number of times on the average at all times of the calibration process.

The calibration process ensures that all the patterns present in the data are defined in a meaningful coordinate system, and this is why the Kohonen neural network is often called a self-organized map. The Kohonen neural network reduces the dimension of a problem, from an $n$-dimension input vector to 2-dimension solution, so as to produce a clearer view of the data patterns (Kohonen, 1990).

\subsection{Predictive neural networks}

As a predictive tool, the multilayer perceptron network with biases, an input layer, a single hidden sigmoid layer, and a linear output layer is by far the most commonly used network topology in the field of water resources (Coulibaly et al., 1999; Maier and Dandy, 2000). They are able to approximate any function with a finite number of discontinuities (Cybenko, 1989; Hornik et al., 1989), provided that the training is adequate. The Levenberg-Marquardt backpropagation algorithm (Hagan and Menhaj, 1994), a second-order nonlinear optimization technique, is selected for the calibration or training of the network weights, because this technique is usually faster and more reliable than any other backpropagation variants (Tan and Van Cauwenberghe, 1999).

Generalization, which is the ability to provide accurate output values for input values that have never been seen by the network, is achieved by the combination of two complementary approaches. The first approach, Bayesian regularization, relates to the training procedure. It involves a multiple objective optimization by which both the sum of the squared errors and the sum of the squared weights must be minimized (MacKay, 1992; Foresee and Hagan, 1997). Bayesian regularization is particularly suited to reduce variance errors, because the minimization constrains the weight to small values, making less likely the possibility of large fluctuations in the response of the network given inputs of large magnitude. An application of this approach in hydrology can be found in Anctil et al. (2004a). The second approach, bagging (Breiman, 1996), relates to techniques used for constructing training data sets. Several training data sets are created from the original data set by bootstrap, which is random picking with replacement. Each of these training sets is employed to train a neural network. Hence a pool of models is created, and a global predictor can be obtained by the mean of their estimates for a given input vector. It has been demonstrated that bagging can reduce variance errors, since aggregation has the effect of smoothing fluctuations from the estimates of all the models (Breiman, 1996, 2001). An application of this approach in hydrology can be found in Cannon and Whitfield (2002).

Predictive neural networks are developed for one-day ahead streamflow forecasts, and predictive performance is globally assessed in this application by the sum of squared errors:

$\mathrm{SSE}=\sum_{t=1}^{n}\left(Q_{\mathrm{obs}, t+L}-Q_{\mathrm{est}, t+L}\right)^{2}$

the root mean squared errors:

$\operatorname{RMSE}=\left(\frac{\mathrm{SSE}}{n}\right)^{0.5}$

and the persistence index (Kitanidis and Bras, 1980):

PERS $=1-\frac{\text { SSE }}{\text { SSE }_{\text {naive }}}$

where $\mathrm{SSE}_{\text {naive }}$ is a scaling factor expressed as:

$\operatorname{SSE}_{\text {naive }}=\sum_{t=1}^{n}\left(Q_{\mathrm{obs}, t+L}-Q_{\mathrm{obs}, t}\right)^{2}$

In Eq.(3), $Q_{\text {est }, t}$ is the neural network forecast of the observed streamflow $Q_{\text {obs }, t}$ at time step $t$ where $t=1,2, \ldots, n, L$ is the lead time ( $L=1$ for one day-ahead forecast), and $n$ is the number of time steps where model error can be calculated. A PERS value of 1 reflects a perfect fit between predicted and observed values, but 0 is reached when $\mathrm{SSE}=\mathrm{SSE}_{\text {naive }}$, which is equivalent to saying that the rainfall-runoff model is no better than the naïve model. PERS statistics are particularly well suited for assessing forecasts, considering that the previous streamflow is usually one of the neural network input vectors. Negative PERS values would thus signify that the model is degrading the provided information.

\section{Context of application}

\subsection{The Bas-en-Basset watershed}

This study focuses on the Bas-en-Basset watershed, $3234 \mathrm{~km}^{2}$, located in the Western Mediterranean region, in Southern France. Figure 1 provides a schematic of this watershed. Its main stream is actually the upstream reach of the Loire River, encased in mountain formations that separate the large hydrographic systems of the Loire, Rhone, and Garonne rivers. The Western Mediterranean region is an interesting domain for the study of precipitation fields, because the climate is prone to high precipitation rates, such as daily rainfalls in excess of $200 \mathrm{~mm}$. This is especially 


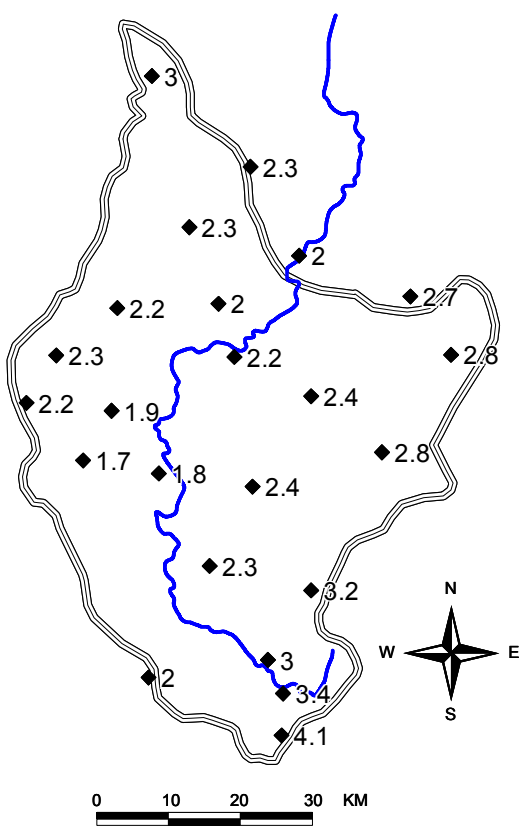

Fig. 1. Schema of the Bas-en-Basset watershed, with precipitation average at each of the rain gauges (including non-rainy days).

true during fall when the Mediterranean Sea surface temperatures are still high from the summer heating while the onset of fall increases the chances of strong synoptic forcing. Convection also plays an important role in a good number of these events, mostly from the Mediterranean Sea itself and the complex terrain features surrounding it. Detailed analyses of some major Western Mediterranean rainstorms are provided by Sénési et al. (1996), Doswell et al. (1998), and Bechtold and Bazile (2001), some of which lead to flash floods that caused fatalities and large property damage.

Daily streamflow is observed at the Bas-en-Basset watershed outlet, and a total of 23 rain gauges, identified by diamonds in Fig. 1, have been available for the observation of daily rainfall from 1980 to 2000 . For the purpose of the clustering of precipitations fields, only the days with no missing observations in the set of rain gauges are considered. A total of 5100 days are thus available for the application, 3931 of which being days when precipitation is observed at one or more rain gauges. The numbers in Fig. 1 represent the average daily precipitation at each rain gauge, calculated with the 5100 available days.

\subsection{Protocol of experiment}

In the first step, the clustering of the daily precipitation fields is performed using Kohonen neural networks. Several initial tests have been conducted to cluster the precipitation fields, with the number of evaluated clusters or groups set between 2 and 12 groups. The number of available data records is the major constraint limiting the number of groups that can be defined. For this application, the 3- and 6-group clustering are deemed adequate, being small enough to offer an easy analysis while being large enough to allow a good discrimination of precipitation fields. The input vectors for the Kohonen network are composed of the daily observations at each of the 23 rain gauges. Prior to being fed to the network, the input vectors are normalised on a daily basis. Hence, a given vector provides the daily precipitation observations, minus the daily precipitation average, divided by the daily standard deviation. This normalisation ensures that all input vectors are on the same scale while preserving the spatial distribution of the daily precipitation fields, which is the feature that is discriminated in the clustering.

In the second step, multilayer perceptron neural networks are trained for the prediction of one-day ahead streamflow values for the Bas-en-Basset watershed. The method for the construction of training and validation sets proposed here makes use of the Kohonen clustering of precipitation fields. The random selection process is structured so that, for any given group of precipitation fields, two-thirds of the associated input vectors go for training and the other third is assigned for validation. This two-thirds/one third ratio selection is accomplished with the 6-group clustering, and the selection is such that the ratio is also respected for the 3-group clustering. A 1-group clustering, where rainy days are all gathered in one single group, is considered and used in the results section as a reference. This approach implies that the validation data set is also used for model selection.

Three input vector configurations, which are designed to define the heterogeneity of precipitation fields, are tested. All configurations use streamflow at day $t$ for the prediction of streamflow at day $t+1$. The first configuration, which is the reference, uses the areal mean precipitation at day $t$ (i.e., all 23 rain gauges) as an input. In the second configuration, the watershed is subjectively divided into two regions based on the clustering of precipitation fields, and the mean areal precipitation of each of these regions is employed as inputs. In the third configuration, the watershed is subjectively divided into four regions, yielding four areal mean precipitation inputs. The performance of the neural networks is analysed with respect to each group of precipitation fields for each clustering (1-, 3- and 6-group).

\section{Results}

\subsection{Clustering of precipitation fields}

The daily mean precipitation at the rain gauges presented in Fig. 1, over the whole database and regardless of any clustering, indicates higher precipitation in the north and the south of the watershed, due to orographic effects. A figure illustrating the standard deviation instead of the mean would show the same spatial heterogeneity as a result of the mountains in the north and south of the watershed. There may be several 


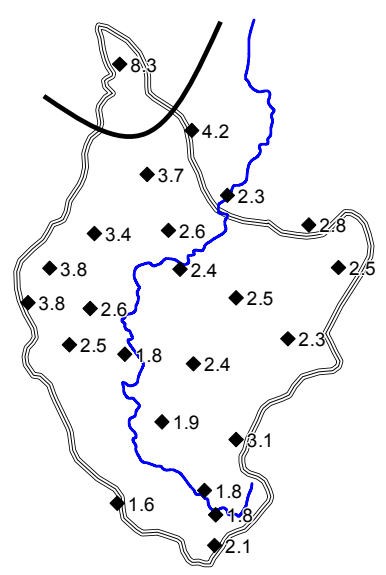

(a)

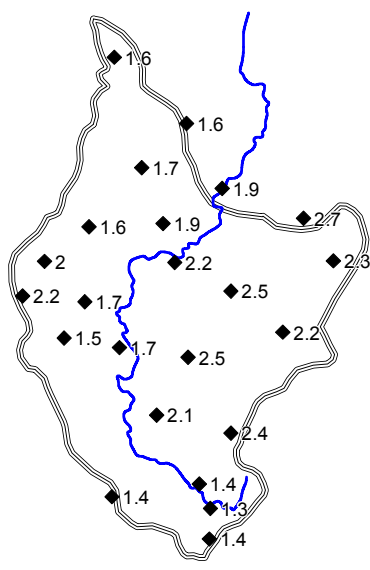

(b)

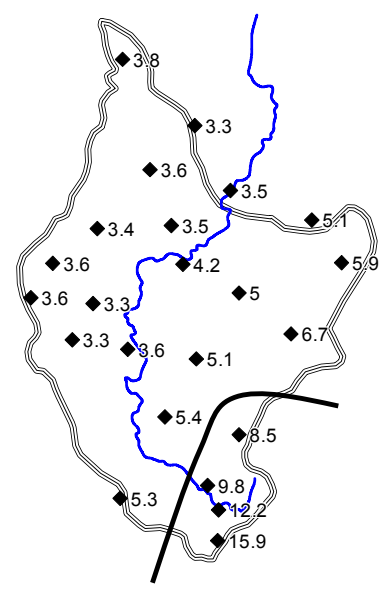

(c)

Fig. 2. Average daily precipitation at every rain gauge, per group, for the clustering of precipitation fields into 3 groups. The thick line illustrates a subjective division of the watershed.

types of heterogeneous precipitation events on this watershed, and the goal of the clustering applied here is to identify them from day to day.

The clustering into three groups (see Fig. 2) fulfills expectations on the aspect of the discrimination of precipitation fields. Similar in format to Fig. 1, Fig. 2 gives the daily mean precipitation at the rain gauges on rainy days, for each group of precipitation fields. Group 3a (Fig. 2a) contains the daily events with high precipitation observed in the northern part of the watershed as a result of the orographic effect. The precipitation cells during these events appear firmly located in the north, for little precipitation is observed in the south, including in the southern mountainous part. Group $3 c$ (see Fig. 2c) includes the daily events with heavy precipitation in the southern part of the watershed. In all likelihood, this group gathers the precipitation events originating from the Mediterranean Sea and the events of lumped heavy precipitation, as demonstrated by the high daily mean at all rain gauges on the watershed. As for group $3 b$ (see Fig. 2b), it represents the daily low precipitation events that are relatively homogeneous spatially. The daily mean precipitation is rather similar from one rain gauge to another and the standard deviations are low as well, and this is indicative of a low spatial variability for the events of this group. The results of the clustering into three groups are satisfying in that it produces groups of precipitation fields that are expected for the Bas-en-Basset watershed. There is one group for the northern orographic effect, another for the southern orographic effect and heavy precipitation overall, and one last group for the relatively low and homogeneous precipitation events. On a hydrologic standpoint, it is likely that each of these groups generates a distinct response from the watershed, and this is partly highlighted by the analysis of the rainfall-runoff relationship.
Table 1. Discrimination of precipitation fields with respect to the clustering into 3 and 6 groups.

\begin{tabular}{cccccccc}
\hline 3-group & \multicolumn{7}{c}{ 6-group clustering } \\
clustering & $6 \mathrm{a}$ & $6 \mathrm{~b}$ & $6 \mathrm{c}$ & $6 \mathrm{~d}$ & $6 \mathrm{e}$ & $6 \mathrm{f}$ & Total \\
\hline 3a & 270 & 3 & 0 & 815 & 249 & 0 & 1337 \\
3b & 299 & 525 & 1 & 4 & 337 & 233 & 1399 \\
3c & 1 & 101 & 755 & 0 & 2 & 336 & 1195 \\
Total & 570 & 629 & 756 & 819 & 588 & 569 & 3931 \\
\hline
\end{tabular}

The results of the clustering into six groups confirm those of the clustering into three groups while refining the discrimination of the daily precipitation events. Figure 3 is similar to Figs. 1 and 2, and summarizes the results of the clustering into six groups. Some groups are typical and already observed in the clustering into three groups. There is one group for the heavy precipitation events due to the mountains in the northern part of the watershed (Fig. 3d), and another one for heavy events due to the mountains and the Mediterranean climate in the southern part of the watershed (Fig. $3 \mathrm{c}$ ). Groups $6 \mathrm{c}$ and $6 \mathrm{~d}$ (Figs. 3c and d) of the six group clustering include the most extreme precipitation events and therefore the most heterogeneous in all likelihood.

As an indication of the agreement between the two clustering, Table 1 gives the distribution of the daily precipitation events following the clustering into three and six groups. It is noted that the majority of the events in groups $6 \mathrm{c}$ and $6 \mathrm{~d}$ are respectively located in groups $3 \mathrm{c}$ and $3 \mathrm{a}$. Mostly, for all of the groups of the 6-group clustering, the events are distributed either into groups $6 \mathrm{a}$ and $6 \mathrm{~b}$ or into groups $3 \mathrm{~b}$ and $3 \mathrm{c}$ in the 3 -group clustering. There is no distribution into groups $3 \mathrm{a}$ and $3 \mathrm{c}$ from any group of the 6-group clustering. 


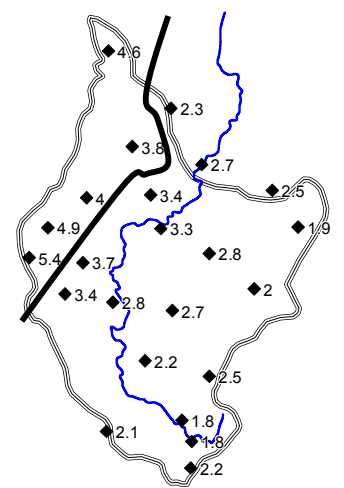

(a)

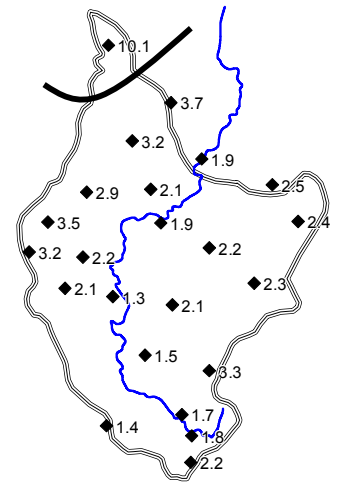

(d)

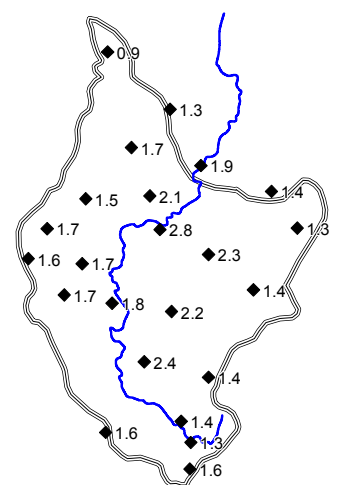

(b)

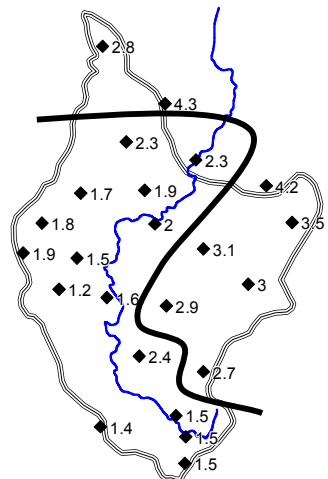

(e)

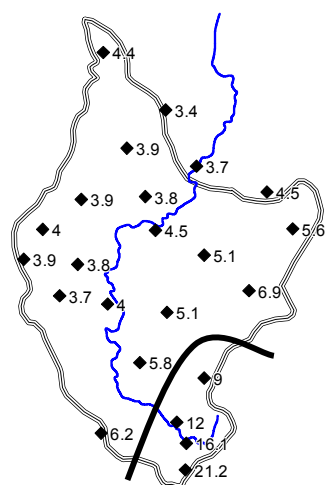

(c)

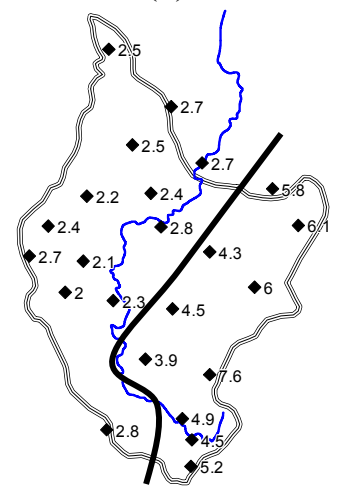

(f)

Fig. 3. Average daily precipitation at every rain gauge, per group, for the clustering of precipitation fields into 6 groups. The thick line illustrates a subjective division of the watershed.
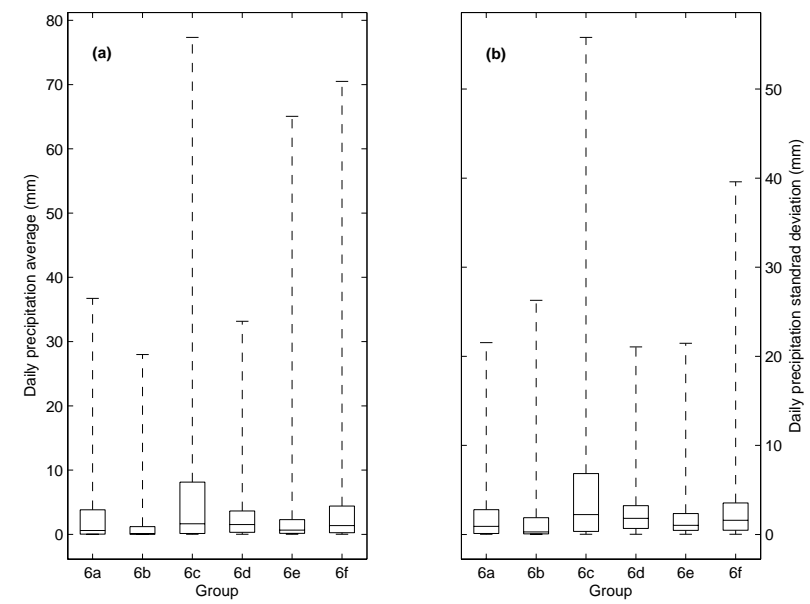

Fig. 4. Box and whisker plot of the distribution of the daily mean precipitation (a) and daily standard deviation of precipitation (b) computed from all 23 rain gauges with respect to each 6-group clusters. The boxes have lines at the lower quartile, median, and upper quartile values. The whiskers are lines extending from the minimum value to the maximum value.
Groups 6e and 6f (Figs. 3e and f) show not only a north-south variation in the location of the precipitation cell, but also an east-west variation as well, which cannot be noticed in the 3 -group clustering. Groups $6 \mathrm{a}$ and $6 \mathrm{~b}$ (Figs. 3a and b) should be considered as containing relatively low and homogeneous precipitation events.

As a general rule for this watershed, the heavier is the precipitation event, the more heterogeneous it is susceptible to be. This is confirmed by Fig. 4, which show the distribution of the (a) mean and (b) standard deviation of the precipitation events according to each group for the 6-group clustering. Similar results are obtained for the 3-group clustering. There is a clear distinction between group $6 \mathrm{c}$, which contains the heaviest and most heterogeneous daily precipitation events, and all the other groups. The events of group $6 \mathrm{c}$ are very likely to produce a response in terms of streamflow production that may significantly differ from that of the other groups. The other groups may well each generate a distinct response from the watershed, although it might not be easy to clearly distinguish each one from the others. Assessing the response of the watershed to precipitation events is implicitly accomplished here through the analysis of the performance of rainfall-runoff models with respect to the groups identified in both clustering. 


\subsection{Rainfall-runoff relationship}

Pertinent input vectors for Bas-en-Basset one day-ahead streamflow forecasts are the streamflow and the precipitation of the previous day. These have been identified in a step-wise manner, as in Anctil et al. (2004b), from a pool of candidates consisting of streamflow, mean areal rainfall and potential evapotranspiration with time-lags of one to three days, arbitrarily fixing the number of hidden neurons to 5. A more "physical approach determining lags may have relied on the travel time through the watershed. The goal of input selection is to maximize the PERS for the validation dataset (one third of the database), while the network weights are optimized for the training dataset (two thirds of the database). After the input selection, the number of hidden neurons is optimized by trial and error from 2 to 35. The final network architecture and the number of parameters (weights and drifts) to calibrate for each of the models are given in Table 2.

The performance of the proposed network relies heavily on the high autocorrelation of flow, so that the current flow mainly depends on the flow of the previous day. Any measurement errors of the current flow will reflect strongly on the forecasting ability of the network.

As for the use of precipitation inputs, three cases are considered. The first case employs the areal mean precipitation, calculated with all 23 rain gauges. The other two cases subjectively divide the watershed into 2 and 4 regions, respectively, and the mean precipitation of each of the regions are used as inputs. Figure 5 illustrates these regions. In case 2 (2 mean precipitation inputs), the southern region includes the rain gauges with the heaviest precipitation measurements observed. In case 3 (4 mean precipitation inputs), the regions are set to account for both the north-south and eastwest precipitation field variability observed during the clustering. Case 1 represents the reference while cases 2 and 3 constitute attempts to improve rainfall-runoff modelling performance. One rainfall-runoff model is developed for each case, and their performance is summarized in Table 2 with respect to SSE, RMSE and PERS. The results show that increasing the number of precipitation inputs does not lead to improved performance. A priori, performance decreases as the network becomes less parsimonious, although a final conclusion can only be made after modelling performance is analyzed with respect to the groups of precipitation fields.

Table 3 details model performance with respect to both the modelling cases (1, 2 or 4 precipitation inputs) and the groups from Kohonen clustering, for all three performance indicators (SSE, RMSE and PERS). The best modelling scenarios are those that yield an SSE and a RMSE that are closer to 0 and a PERS that is closer to 1 . The situation where no clustering is performed, gathering all precipitation fields in one single group, is also given in Table 3 for comparison. Note that group 0 represents the days when no precipitation is recorded at any of the rain gauges.

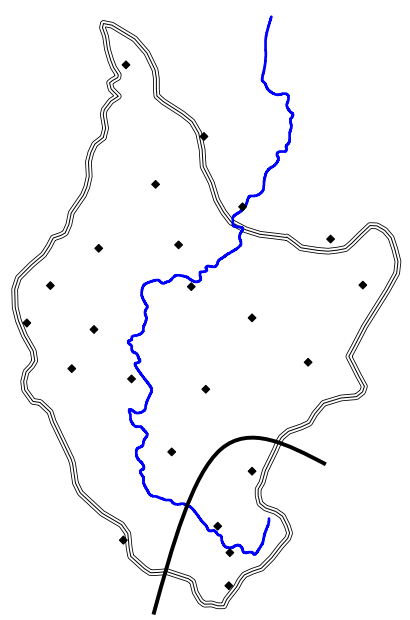

(a)

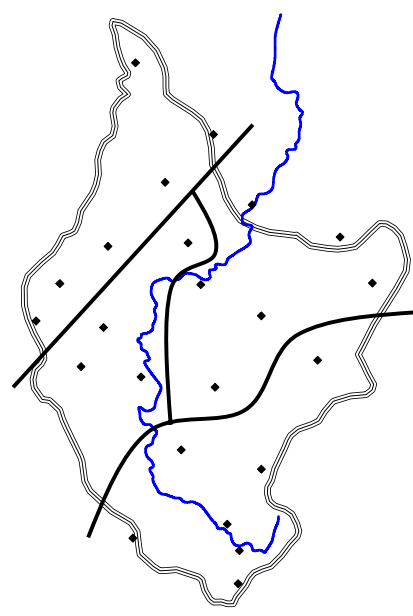

(b)
Fig. 5. Division of the watershed into (a) two and (b) four regions for the determination of precipitation inputs for the rainfall-runoff models.

In terms of the SSE, groups 3c and 6c (i.e., southern orography and heavy precipitation) are the highest, with group $3 \mathrm{a}$ and group $6 \mathrm{~d}$ (i.e., northern orography) possessing the second largest SSE on most occasions. The RMSE obviously confirms the conclusion about the SSE, as it translates the sum of errors into an average error associated to a single day. However, SSEs and RMSEs must be weighted with respect to the amount of precipitation and streamflow level (e.g. groups $3 \mathrm{c}$ and $6 \mathrm{c}$ ), as they usually become larger as the average precipitation and streamflow increase.

Larger SSEs and RMSEs do not necessarily translate into poor performance in terms of PERS. The neural network models for the situation involving groups $3 \mathrm{c}$ and $6 \mathrm{c}$ precipitation fields possess large SSE and RMSE compared with the other groups, but also a large PERS compared with the other groups. It indicates that neural networks are far better alternative than the reference model for PERS (i.e., the naïve model) in the cases of highly heterogeneous precipitation fields, compared with cases of relatively homogeneous precipitation fields or cases where no precipitation is observed. It is expected, for any hydrological model, that large errors are produced in the events of large streamflow or precipitation.

The use of more than one precipitation input, either 2 or 4 , has been justified by the expectation of improving modelling performance, although the results in Table 3 do not show this to be the case. Deterioration occurs for the more heterogeneous precipitation fields, namely groups $3 \mathrm{a}$ and $3 \mathrm{c}$, and groups $6 \mathrm{a}, 6 \mathrm{c}, 6 \mathrm{~d}$ and $6 \mathrm{f}$. In the other cases, improvement is only marginal. The definition of good inputs is critical for modelling performance, and the division into regions as performed here has been subjective. The use of a more objective approach, based on a systemic exploration of the 
Table 2. Summary of performance for the three cases of rainfall-runoff modelling according to precipitation inputs.

\begin{tabular}{cccccc}
\hline $\begin{array}{c}\text { Number of } \\
\text { precipitation inputs }\end{array}$ & $\begin{array}{c}\text { Network } \\
\text { architecture }\end{array}$ & $\begin{array}{c}\text { Number of } \\
\text { parameters }\end{array}$ & $\begin{array}{c}\text { SSE } \\
\left(\mathrm{mm}^{2}\right)\end{array}$ & $\begin{array}{c}\text { RMSE } \\
(\mathrm{mm})\end{array}$ & PERS \\
\hline 1 & $2-7-1$ & 29 & 171.7 & 0.316 & 0.795 \\
2 & $3-6-1$ & 31 & 179.5 & 0.323 & 0.785 \\
4 & $5-5-1$ & 36 & 195.3 & 0.337 & 0.767 \\
\hline
\end{tabular}

Table 3. Summary of rainfall-runoff modelling performance per group of precipitation fields, exploiting the network architectures identified in Table 2.

\begin{tabular}{|c|c|c|c|c|c|c|c|c|c|}
\hline \multirow{2}{*}{$\begin{array}{l}\text { Group } \\
\text { letter }\end{array}$} & \multicolumn{3}{|c|}{ No clustering } & \multicolumn{3}{|c|}{ 3-group clustering } & \multicolumn{3}{|c|}{ 6-group clustering } \\
\hline & $\begin{array}{c}\text { SSE } \\
\left(\mathrm{mm}^{2}\right)\end{array}$ & $\begin{array}{c}\text { RMSE } \\
(\mathrm{mm})\end{array}$ & PERS & $\begin{array}{c}\text { SSE } \\
\left(\mathrm{mm}^{2}\right)\end{array}$ & $\begin{array}{l}\text { RMSE } \\
(\mathrm{mm})\end{array}$ & PERS & $\begin{array}{c}\text { SSE } \\
\left(\mathrm{mm}^{2}\right)\end{array}$ & $\begin{array}{l}\text { RMSE } \\
(\mathrm{mm})\end{array}$ & PERS \\
\hline \multicolumn{10}{|c|}{ One precipitation input } \\
\hline 0 & 5.2 & 0.112 & 0.680 & 5.2 & 0.112 & 0.680 & 5.2 & 0.112 & 0.680 \\
\hline $\mathrm{a}$ & 166.6 & 0.357 & 0.797 & 19.6 & 0.215 & 0.704 & 4.0 & 0.155 & 0.718 \\
\hline $\mathrm{b}$ & & & & 22.5 & 0.215 & 0.809 & 4.4 & 0.140 & 0.559 \\
\hline $\mathrm{c}$ & & & & 124.5 & 0.559 & 0.804 & 117.1 & 0.671 & 0.810 \\
\hline $\mathrm{d}$ & & & & & & & 15.6 & 0.237 & 0.705 \\
\hline $\mathrm{e}$ & & & & & & & 17.3 & 0.303 & 0.835 \\
\hline $\mathrm{f}$ & & & & & & & 8.1 & 0.206 & 0.656 \\
\hline \multicolumn{10}{|c|}{ Two precipitation inputs } \\
\hline 0 & 5.2 & 0.113 & 0.675 & 5.2 & 0.113 & 0.675 & 5.2 & 0.113 & 0.675 \\
\hline $\mathrm{a}$ & 174.2 & 0.365 & 0.788 & 20.5 & 0.219 & 0.690 & 4.8 & 0.169 & 0.664 \\
\hline $\mathrm{b}$ & & & & 14.4 & 0.172 & 0.878 & 4.8 & 0.147 & 0.513 \\
\hline $\mathrm{c}$ & & & & 139.3 & 0.591 & 0.781 & 131.2 & 0.710 & 0.787 \\
\hline $\mathrm{d}$ & & & & & & & 15.6 & 0.237 & 0.705 \\
\hline $\mathrm{e}$ & & & & & & & 9.5 & 0.224 & 0.909 \\
\hline $\mathrm{f}$ & & & & & & & 8.2 & 0.208 & 0.649 \\
\hline \multicolumn{10}{|c|}{ Four precipitation inputs } \\
\hline 0 & 5.0 & 0.110 & 0.692 & 5.0 & 0.110 & 0.692 & 5.0 & 0.110 & 0.692 \\
\hline a & 190.3 & 0.381 & 0.768 & 22.1 & 0.228 & 0.667 & 5.9 & 0.187 & 0.587 \\
\hline $\mathrm{b}$ & & & & 16.4 & 0.184 & 0.860 & 3.5 & 0.125 & 0.646 \\
\hline $\mathrm{c}$ & & & & 151.9 & 0.617 & 0.761 & 144.4 & 0.745 & 0.765 \\
\hline $\mathrm{d}$ & & & & & & & 18.0 & 0.254 & 0.661 \\
\hline $\mathrm{e}$ & & & & & & & 9.1 & 0.219 & 0.913 \\
\hline $\mathrm{f}$ & & & & & & & 9.4 & 0.222 & 0.599 \\
\hline
\end{tabular}

Note: the 0 group represent the days when no precipitation is recorded at any of the rain gauges.

combinations of rain gauges available for the calculation of areal mean precipitation, is recommended here. An alternative approach would be trying to group the points based on the corresponding travel time thus creating groups of gauges with similar influence on the flow downstream. In the present study, however, the available data does not have a high enough temporal resolution to investigate this idea. Another alternative for modelling improvement would be to develop a distinct model for each of the groups, which is feasible if the database is large enough to accommodate this community of models. The preliminary tests performed on this watershed indicate that the available database needs to be larger, as no significant improvement is noticed. In the present situation, input parsimony is advantageous to the performance of the networks.
With respect to the advantage of multilayer perceptron neural networks, the results demonstrate that they have a capacity to accommodate heterogeneous precipitation fields. The training process may generate a network topology that can distinguish between precipitation events, even if only two inputs, streamflow and mean areal precipitation, are given. In the case of Bas-en-Basset, it can be assumed that two significantly different precipitation fields exist, that is, those in groups $3 \mathrm{c}$ and $6 \mathrm{c}$ (southern orography and heavy precipitation) and all the others combined (Fig. 4). These two significant precipitation fields can easily be distinguished by the amount of precipitation involved (e.g., smaller or bigger than a given threshold), and this is why only one precipitation input may be enough to differentiate between these two fields in the Bas-en-Basset watershed. In the situation where 
more than two precipitation fields are present, more than two precipitation inputs would be necessary to give the network topology a chance to make a distinction among the fields.

\section{Conclusion}

The goal of this work has been to accomplish a clustering of precipitation fields so as to distinguish between homogeneous and heterogeneous precipitation events and hence collect information to better support rainfall-runoff modelling efforts on the Bas-en-Basset watershed from which the precipitation data come from. Kohonen neural networks are used as the clustering tool while multilayer preceptron neural networks are employed as lumped models for one-day ahead streamflow prediction. The results of the clustering validate the use of the Kohonen network as a classifier of precipitation fields. The clustering generates groups of precipitation fields that are expected to exist on the Bas-en-Basset watershed considering the general climate patterns of the region and the physical constraints (orography). The clustering can help afterwards to refine the analysis of the performance of rainfall-runoff models. Performance can be analysed with respect to each group of precipitation fields. The performance analysis accomplished on the Bas-en-Basset watershed has shown that rainfall-runoff models produce the largest errors for cases of moderately to highly heterogeneous precipitation fields, which is to be expected of conventional hydrological models. On the basis of this analysis with respect to group of precipitation fields, solutions such as the addition of precipitation inputs or the development of specific models per group can be envisioned. The use of more than one precipitation input has not led to performance improvement in rainfall-runoff modelling for this application. With respect to the advantage of multilayer perceptron neural networks, it can be said that they can account for heterogeneous precipitation, providing that enough inputs are given to the models to allow for the distinction between the existing precipitation fields. In the case of the Bas-en-Basset watershed, it can be assumed that only two significantly distinct precipitation fields actually exist, and consequently only one precipitation input is required. Further development on this application would involve the exploration of combinations of rain gauges from among the 23 available that would be better able to represent precipitation on the watershed and lead to improved rainfall-runoff modelling performance.

Acknowledgements. The authors wish to acknowledge the financial contribution for this project of the Natural Science and Engineering Council of Canada (NSERC) and of the Fonds québecois de recherche sur la nature et les technologies (FQRNT).

Edited by: A. Montanari

\section{References}

Anctil, F., Perrin, C., and Andréassian, V.: ANN output updating of lumped conceptual rainfall-runoff forecasting models, J. Am. Water Resour. Assoc., 39(5), 1269-1279, 2003.

Anctil, F., Perrin, P., and Andréassian, V.: Impact of the length of observed records on the performance of ANN and of conceptual parsimonious rainfall-runoff forecasting models, Environ. Modelling and Software, 19, 357-368, 2004a.

Anctil, F., Michel, C., Perrin, C., and Andréassian, V.: A soil moisture index as an auxiliary ANN input for stream flow prediction, J. Hydrol., 286, 155-167, 2004b.

ASCE: Artificial neural networks in hydrology - I: Preliminary concepts, ASCE J. Hydrol. Eng., 5(2), 115-123, 2000a.

ASCE: Artificial neural networks in hydrology - II: Hydrologic applications, ASCE J. Hydrol. Eng., 5(2), 124-137, $2000 \mathrm{~b}$.

Bardossy, A. and Plate, E. J.: Space-time model for daily rainfall using atmospheric circulation patterns, Water Resour. Res., 28(5), 1247-1259, 1992.

Bardossy, A., Muster, H. Duckstein, L., and Bogardi, I.: Knowledge-based classification of circulation patterns for stochastic precipitation modelling, Proc. Stochastic and Statist Methods in Hydrol. Environ. Eng., Waterloo, Ontario, Canada, 21-23 June 1993, 1994.

Bechtold, P. and Bazile, E.: The 12-13 November 1999 flash flood in southern France, Atmos. Res., 56, 171-189, 2001.

Benzie, R., Deidda, R., and Marrocu, M.: Characterization of temperature and precipitation fields over Sardinia with principal component analysis and singular spectrum analysis, Int. J. Climatol., 17(11), 1231-1262, 1997.

Bowden, G. J., Maier, H. R., and Dandy, G. C.: Optimal division of data for neural network models in water resources applications, Water Resour. Res., 38(2), 2.1-2.11, 2002.

Brath, A., Montanari, A., and Toth, E.: Neural networks and non-parametric methods for improving realtime flood forecasting through conceptual hydrological models, Hydrol. Earth Syst Sci., 6(4), 627-640, 2002.

Breiman, L.: Bagging predictors, Machine Learning, 24(2), 123 140, 1996.

Breiman, L.: Using iterated bagging to debias regressions, Machine Learning, 45, 262-277, 2001.

Cannon, A. J. and Whitfield, P. H.: Downscaling recent streamflow conditions in British Columbia, Canada using ensemble neural network models, J. Hydrol., 259, 136-151, 2002.

Coulibaly, P., Anctil, F., and Bobée, B.: Prévision hydrologique par réseaux de neurones artificiels: état de l'art, Can. J. Civil Eng., 26, 293-304, 1999.

Cybenko, G.: Approximation by superposition of a sigmoidal function, Mathematics of Control, Signals and Systems, 2, 303-314, 1989.

Dawdy, D. R. and Bergman, J. M.: Effect of rainfall variability on streamflow simulation, Water Resour. Res., 5(3), 958-966, 1969.

Dillon, W. R. and Goldstein, M.: Multivariate analysis: Methods and applications, John Wiley \& Sons, New York, NY, USA, 1984.

Doswell III, C. A., Ramis, C., Romero, R., and Alonso, S.: A diagnostic study of three heavy precipitation episodes in the western Mediterranean, Weather Forecast, 13, 102-124, 1998.

Faures, J. M., Goodrich, D. C., Woolhiser, D. A., and Sorooshian, S.: Impact of small-scale rainfall variability on runoff simulation, 
J. Hydrol., 173, 309-326, 1995.

Foresee, F. D. and Hagan, M. T.: Gauss-Newton approximation to Bayesian learning, Proceedings, 1997 IEEE Int. Conf. Neural Networks, Houston, TX, 3, 1930-1935, 1997.

Gotz, R., Steiner, B., Sievers, S., Friesel, P., Roch, K., Schworer, R., and Haag, F.: Dioxin, dioxin-like PCBS and organotin compounds in the River Elbe and the Hamburg Harbour: Identification of sources, Water Sci. Technol., 37(6-7), 207-215, 1998.

Hagan, M. T. and Menhaj, M.: Training feedforward networks with the Marquardt algorithm, IEEE Trans. Neural Networks, 5, 989993, 1994.

Hall, M. J. and Minns, A. W.: The classification of hydrologically homogeneous regions, Hydrol. Sci. J., 44(5), 693-704, 1999.

Hornik, K., Stinchcombe, M., and White, H.: Multilayer feedforward networks are universal approximators, Neural Networks, 2, 359-366, 1989.

Hughes, J. P., Lettenmaier, D. P., and Guttorp, P.: A stochastic approach for assessing the effects of changes in synoptic circulation patterns on gauge precipitation, Water Resour. Res., 29(10), 3303-3315, 1993.

Kitadinis, P. K. and Bras, R. L.: Real-time forecasting with a conceptual hydrologic model: 2. Applications and results, Water Resour. Res., 16(6), 1034-1044, 1980.

Kohonen, T.: The self-organizing map, Proc. IEEE, 79(9), 14641480, 1990.

Lauzon, N.: Water Resources Data Quality Assessment and Description of Natural Processes Using Artificial Intelligence Techniques, Ph.D. Thesis, The University of British Columbia, Department of Civil Engineering, Vancouver, BC, Canada, 2003.

Liong, S. Y., Lim, W. H., Kojiri, T., and Hori, T.: Advance flood forecasting for flood stricken Bangladesh with a fuzzy reasoning method, Hydrol. Processes, 14(3), 431-448, 2000.

MacKay, D. J. C.: Bayesian interpolation, Neural Computation, 4(3), 415-447, 1992.

Maier, H. R. and Dandy, G. C.: Neural networks for prediction and forecasting of water resources variables: Review of modelling issues and applications, Environ. Modelling and Software, 15, 101-124, 2000.
Naden, P. S.: Spatial variability in flood estimation for large catchments: The exploitation of channel network structure, Hydrol. Sci. J., 37(1), 53-71, 1992.

Ozelkan, E. C., Ni, F., and Duckstein, L.: Relationship between Monthly Atmospheric Circulation Patterns and Precipitation: Fuzzy Logic and Regression Approaches, Water Resour. Res., 32(7), 2097-2103, 1996.

Schnur, R. and Lettenmaier, D. P.: A case study of statistical downscaling in Australia using weather classification by recursive partitioning, J. Hydrol., 212-213(1-4), 362-379, 1998.

Schoof, J. T. and Pryor, S. C.: Downscaling temperature and precipitation: A comparison of regression-based methods and artificial neural networks, Int. J. Climatol., 21(7), 773-790, 2001.

Sénési, S., Bourgeault, P., Chèze, J.-L., Cosentino, P., and Thepenier, R.-M.: The Vaison-La-Romaine flash flood: Mesoscale analysis and predictability issues, Weather Forecast, 11(4), 417442, 1996.

Siew-Yan-Yu, T. O., Rousselle, J., Jacques, G., and Nguyen, V. T. V.: Régionalisation du régime des précipitations dans la région des Bois-francs et de l'Estrie par l'analyse en composantes principales, Can. J. Civil Eng., 25(6), 1050-1058, 1998.

Singh, V. P. and Woolhiser, D. A.: Mathematical modelling of watershed hydrology, ASCE J. Hydrol. Eng., 7(4), 270-292, 2002.

Tan, Y. and Van Cauwenberghe, A.: Neural-network-based d-stepahead predictors for nonlinear systems with time delay, Eng. Applications of Artificial Intelligence, 12, 21-35, 1999.

Toth, E., Brath, A. and Montanari, A.: A comparison of short-term rainfall prediction models for real-time flood forecasting, J. Hydrol., 239, 132-147, 2000.

Wilson, C. B., Valdes, J. B., and Rodriguez-Iturbe, I.: On the influence of spatial distribution of rainfall on storm runoff, Water Resour. Res., 15(1), 321-328, 1979.

Zorita, E. and Storch, H. V.: The analog method as a simple statistical downscaling technique: Comparison with more complicated methods, J. Climate, 12(8), 2474-2489, 1999.

Zorita, E., Hughes, J. P., Lettenmaier, D. P., and Storch, H. V.: Stochastic characterization or regional circulation patterns for climate models diagnosis and estimation of local precipitation, J. Climate, 8(5), 1023-1042, 1995. 